\title{
BEOBACHTUNGEN DER GEBURT DES FRETTCHENS (MUSTELA FURO L.)
}

\author{
von \\ C. NAAKTGEBOREN \\ Zoologisches Laboratorium der Universität Amsterdam
}

\section{EINLEITUNG}

Über die Geburt der Raubtiere ist bisher noch wenig bekannt. Man findet in der Literatur viel weniger Geburtenbeschreibungen von Raubtieren als von anderen Tiergruppen, z.B. Huftieren und Nagetieren (SlijPER, 1960). Die Raubtiere gehören zu den multiparen Säugern, d.h. sie gebären mehrere Junge in einem Wurf, genau wie die grösste Zahl der Nagetiere (NAAKTGEBOREN, 1960). In dieser Arbeit werden die Ergebnisse von drei Geburtenbeobachtungen mitgeteilt. Obwohl also nicht die Rede ist von einer grossen Zahl von Beobachtungen, ist es meiner Ansicht nach jedoch von Bedeutung diese Ergebnisse zu veröffentlichen, weil sie eine Vervollständigung der Arbeit MurR's (1932) darstellen. Die hochträchtigen Tiere erhielt ich vom Institut für Versuchstierzucht T.N.O. zu Zeist, Niederlande. Ich arbeitete mit zwei wiederholtgebärenden oder pluriparen Tieren und mit einem erstgebärenden oder primiparen Tier.

Die einzige Arbeit, worin die Geburt des Frettchens eingehend beschrieben wird, ist von MurR, (1932). Die Geburt vom Iltis (Mustela putorius $L$.) wurde von Herter (1953) beschrieben. Die Arbeit von Murr (1933) enthält keine neueren Beobachtungen als die von Murr (1932). Herter (1959) fasst nur die Ergebnisse von Murr $(1932,1933)$ und von Herter (1953) zusammen. Mit Ausnahme von diesen zwei Arbeiten sind mir keine Beschreibungen der Geburt von irgendeiner Art der Gattung Mustela bekannt. Abbildungen des gebärenden Muttertieres wurden bisher nie veröffentlicht. Die Ergebnisse von MurR (1932) und Herter (1953) habe ich, der Ubersichtlichkeit wegen, an den betreffenden Stellen, zwischen meinen eigenen Beobachtungen zitiert.

\section{METHODIK}

Jedes Tier wurde in einem Käfig von $80 \times 32 \times$ $40 \mathrm{~cm}$ (,Tageskäfig“) gehalten, der durch eine $8 \times$
$12 \mathrm{~cm}$ grosse Offnung mit dem $29 \times 25 \times 20 \mathrm{~cm}$ messenden "Schlafraum" verbunden ist. Das Tier benutzt immer dieselbe Ecke des Tageskäfigs zum Defaezieren und zur Losung von Urin: die „Kotecke“. Der Schlafraum war aus Holz hergestellt und nur oben von einen gläsernen Deckel versehen. Ein schwarzes Tuch diente zum Abschirmen des Tageslichtes. Zur Beobachtung der Geburt konnte das Tuch entfernt werden. Der Tageskäfig war hauptsächlich aus Glas und perforierten Metallwänden hergestellt. Ihr Futter und ihre Getränke erhielten die Tiere immer im Tageskäfig.

Die Tiere wurden am 18/5/'60 erhalten und die Geburten erfolgten am 24/5/'60 und am 1/6/'60. Es war also möglich, die Tiere schon vor der Geburt regelmässig zu beobachten, denn es ist wichtig, dass die Tiere sich an die regelmässige Beobachtung gewöhnen. Während der Geburt wurden auf Kodak tri $X$ Film Aufnahmen gemacht. Mit diesem hochempfindlichen Film ist es möglich ohne Blitzlicht zu photographieren. Die Tiere wurden also nicht durch Blitzlicht erschreckt, was möglicherweise die Geburt hätte beeinflussen können. Die Kamera (Praktica FX2) wurde schon einen Tag vor der Geburt aufgestellt, um das Muttertier an den neuen Gegenstand in ihrer Umgebung zu gewöhnen. Die Kamera stand auf einem Stativ, sodass fast der ganze Schlafraum in das Kamerabild fiel.

\section{EIGENE BEOBACHTUNGEN}

1. Die Vorboten der bevorstehenden Geburt und die Eröffnungsphase

Das Benehmen des hochträchtigen Muttertieres kurz vor der Geburt ist von grösster Bedeutung, weil man daran oft den genaueren Zeitpunkt der Geburt im voraus bestimmen kann. Im allgemeinen habe ich etwa dieselben Vorzeichen gefunden wie MURR (1932): 
Einfallen an den Flanken, reflektorisches Zucken der Rückenmuskulatur, verminderte Fresslust, zunehmende Trägheit, eine Schwellung der Vulva, Haarausfall und eine Abnahme der Körpertemperatur. Nach MURR können diese Vorboten einige Stunden, aber auch einige Tage, vor der Geburt auftreten. Der Haarausfall war bei meinen Tieren I und II sehr stark und fing schon 6 (Tier I) und 4 (Tier II) Tage vor der Geburt an. Tier III zeigte jedoch überhaupt keinen Haarausfall. Am Haarausfall kann man also den genauen Zeitpunkt der Geburt nicht bestimmen. Die ausgefallenen Haare findet man zum Teil im Tageskäfig. Viel mehr Haare findet man jedoch im Nest im Schlafraum. Zur Einrichtung des Nestes hatte das Tier den Wänden des Schlafraums entlang Heu aufgeschichtet und in der Mitte des Raumes den Bodenbelag bis auf das Holz kahlgekratzt. Herter (1953) beschreibt das Nest vom Iltis in derselben Weise. Ich war nicht in der Lage den Nestbau zu beobachten, weil das Heu schon im Schlafraum lag ehe die Tiere zum ersten Mal in ihre Käfige gebracht wurden. Eine starke Abnahme der Fresslust, stark zunehmende Trägheit, oftmals auf dem Rücken Liegen und regelmässiges Lecken der Vulva, der Inguinalgegend, des Bauches und der Zitzen sind Vorboten, die höchstens einen Tag vor der Geburt auftreten. Am letzten Tag vor der Geburt liegt das Tier sehr viel auf dem Rücken im Schlafraum. Im Abdomen beobachtet man gelegentlich regelmässige Kontraktionen (Wehen), die $r$ isch aufeinander folgen. Das Tier keucht dann und wann. Nach einiger Zeit werden die Wehen wieder schwächer und manchmal schläft das Tier ein.

Die Symphysis pelvis verschlafft vor der Geburt nicht, wie dieses z.B. beim Cavia der Fall ist. (Granzow, 1930). Wohl beobachtet man kurz vor der Geburt eine Schwellung der Vulva (MurR, 1932). Ich habe diese Schwellung auch festgestellt. Kurz vor der Geburt tritt eine Schleimschnur aus der Vulva. Ich habe dies 2 bis 4 Stunden vor der Geburt feststellen können bei den Tieren I und II, während dies möglicherweilse bei Tier III auch aufgetreten ist, aber nicht beobachtet wurde, weil meine Aufmerksamkeit völlig auf das gebärende Tier II gerichtet war. Die Schleimschnur ist 0,6 bis $1,5 \mathrm{~cm}$ lang und 1 bis 1,5 $\mathrm{mm}$ dick und besteht aus glasigem Schleim, ohne Beimischung von Blut. Auch Murr hat diese Schleimausscheidung beobachtet. Er spricht von einer Schleimsekretion der Vagina. Meiner Ansicht nach ist es wahrscheinlicher, dass die Schleimschnur, die aus der Vulva tritt, durch Auflösung des zervikalen Schleimpfropfes zustande kommt, wie dieses bei manchen Säugern, wie z.B. beim Rinde, der Fall ist
(SToss, 1944). Wenn der zervikale Schleimpfropf aufgelöst und die Zervix erschlafft ist, ist es möglich, dass die Eröffnungswehen die Zervix völlig eröffnen.

Sehr kurz vor der Geburt werden die Tiere unruhig. Sie wechseln vom Schlafraum in den Tageskäfig über und zurück. Die Tiere trinken sogar in liegender Haltung, was ich besonders bei Tier II mehrere Male beobachtet habe, obwohl auch die beiden anderen Tiere dasselbe Benehmen gezeigt haben. Das Tier II versuchte aufzustehen, was aber nicht gelang, weil das Tier durch die Hinterbeine knickte. Es hat sich also herausgestellt, dass grosse Trägheit, Ausscheidung von einer glasigen Schleimschnur und Schwierigkeiten beim Stehen und Gehen die wichtigsten Merkmale sind zum Feststellen der unmittelbar bevorstehenden Geburt.

Die Dauer der Eröffnungsphase lässt sich nur sehr schwierig feststellen, weil man den Anfang fast niemals genau bestimmen kann. MUrR (1932) sagt, dass dieses Stadium mehrere Stunden in Anspruch nimmt. Er stellte sogar einmal eine Eröffnungsdauer von 18 Stunden fest. Auch ich habe gefunden, dass die Eröfnungsphase viel Zeit beansprucht, und dass man für die Eröffnungsphase mindestens die doppelte bis vierfache Dauer der Austreibezeit annehmen darf.

\section{Die Austreibungsphase}

Die Austreibung der Jungen wurde nur bei den Tieren II und III beobachtet. Ich habe keine Unterschiede zwischen dem pluriparen und dem primiparen Tier feststellen können. Die Austreibung folgt immer auf ein sehr frequentes Lecken der Vulva (Abb. 1), des Bauches, der Zitzen und der Leistengegend. Der ganze Körper wird von zuckenden Bewegungen durchzogen und man kann von aussen sogar die Kontraktionen des Uterus sehen. Diese Befunde gelten für die Austreibungsphase als ganzes, sowie für die Austreibung jeder einzelnen Frucht. Das Tier wird jetzt unruhig und wandert wiederholt vom Schlafraum in den Tageskäfig und wieder zurück. Auch Murn (1932) beschreibt die Unruhe des kurz vor der Geburt stehenden Muttertieres. Es ist von Interesse, dass sich die Tiere vor der Geburt des ersten Jungen in die Kotecke begaben. Die Tiere pressten dann in typischer Kotstellung. Dies wiederholte sich bis in der Vulva das Junge zum Vorschein kam. Tier III zeigte dieses Benehmen auch bei der Geburt des zweiten Jungen, während Tier II sogar für die ersten sechs Jungen in der Kotecke presste. Bei der Geburt ihrer späteren Jungen blieben die Tiere im Schlafraum liegen (Abb. 2). Pressen in typischer Kotstellung ist schon bei manchen Tieren beschrieben worden (SlijPER, 1960). Auch MuRR (1932) 

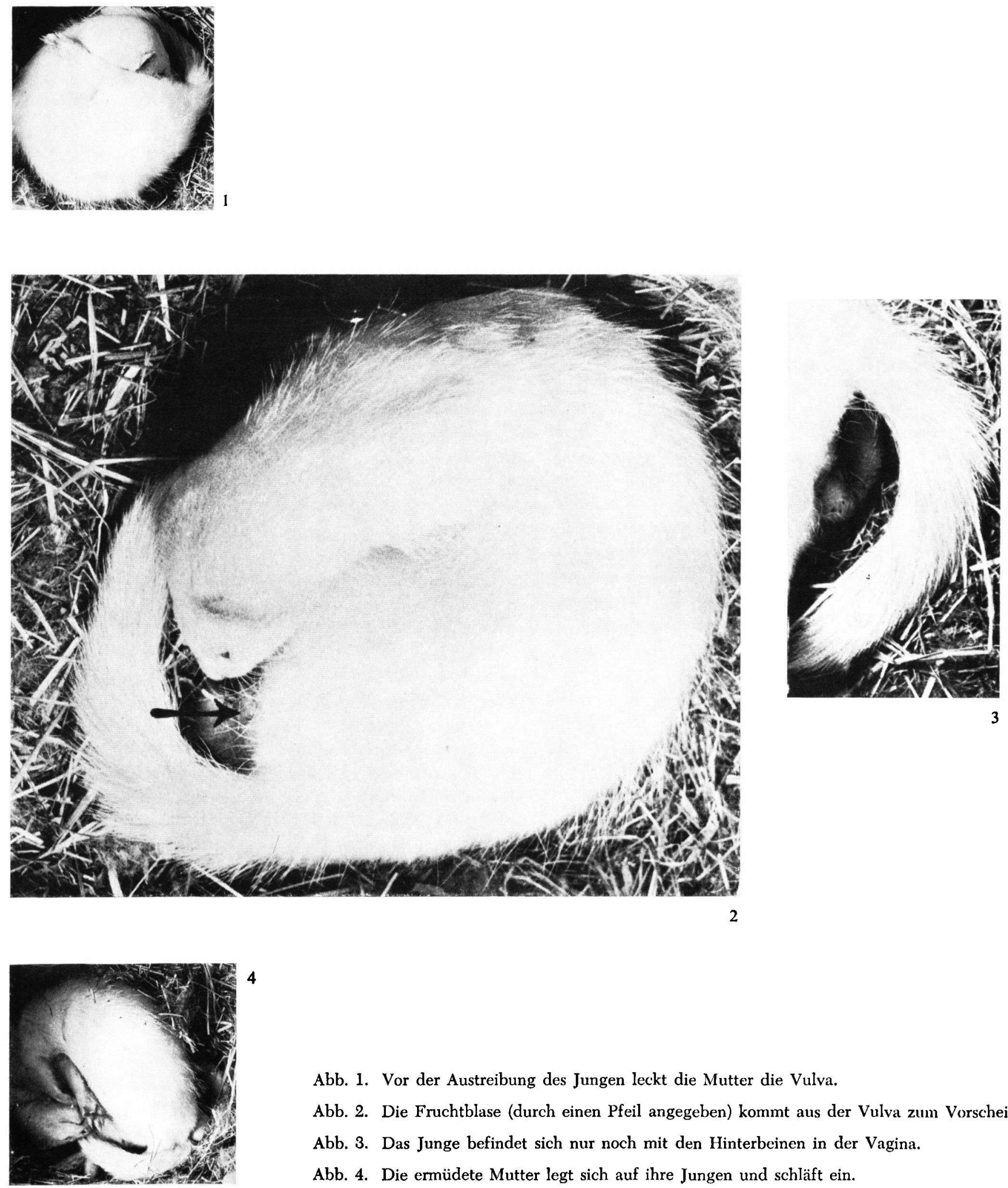

Abb. 1. Vor der Austreibung des Jungen leckt die Mutter die Vulva.

Abb. 2. Die Fruchtblase (durch einen Pfeil angegeben) kommt aus der Vulva zum Vorschein Abb. 3. Das Junge befindet sich nur noch mit den Hinterbeinen in der Vagina. Abb. 4. Die ermüdete Mutter legt sich auf ihre Jungen und schläft ein. 
hat es beim Frettchen beobachtet. Es ist aber sehr interessant, dass die Tiere dazu in die Kotecke gehen. Für das Tier handelt es sich möchlicherweise um einen Reiz, den das Tier nicht vom Kotdrang unterscheiden kann. Nach wiederholtem Pressen tritt die braungelbe Fruchtblase aus der Vulva hervor.

Nur ausnahmsweise findet der Blasensprung statt bevor das Junge teilweise aus der Vulva hängt. Von den 16 Jungen, deren Geburt ich beobachtete, fand nur in 3 oder 4 Fällen ein spontaner Blasensprung statt, bevor das Junge zum Teil geboren war. Nach MURR (1932) können 10 oder mehr Minuten vergehen zwischen Blasensprung und Austreibung des Jungen. Er beschreibt den vor der Austreibung stattfindenden Blasensprung als den meist üblichen Vorgang und erwähnt die Möglichkeit des längeren Intaktbleibens der Fruchthüllen mit keinem Wort. Ich habe aber festgestellt, dass das Muttertier meistens die Fruchtblase mit den Eckzähnen zerreisst, wenn das Junge schon zu einem Viertel oder mehr aus der Vulva hängt. Das siebente Junge von Tier III wurde sogar bis zuletzt in den völlig unversehrten Fruchthüllen ausgetrieben. Mit dem teilweise aus der Vulva hängenden Jungen, geht die Mutter in den Schlafraum und legt sich auf die Seite, oder gelegentlich auf den Rücken oder auf den Bauch. MurR (1932) sagt, dass die Tiere meistens auf dem Rücken oder auf dem Bauch liegen und HerTer (1953) beobachtete, dass eine Iltisfähe auf dem Bauch lag. Die Mutter leckt das Junge und presst es jetzt im Wehenrhythmus nach aussen, was mehrere Minuten dauern kann (Abb. 3). Oftmals war der Schwanz der Mutter stark dorsal gerichtet, wodurch ein grösserer Raum im mütterlichen Becken entsteht (Abb. 5), was für eine schnelle und glatte Austreibung von grösster Bedeutung ist. Das Junge wird also nur durch die Wehen, die von der Bauchpresse unterstützt werden, ausgetrieben. Murr $(1932,1933)$ beschreibt die „,natürliche zangengeburt". Die Mutter fasst das Junge zwischen den Zähnen und zieht es im Wehenrhythmus aus der Vulva. Auch Herter (1953) beschreibt dieses Benehmen, aber die Iltismutter zog ihre Jungen mit einem Ruck aus der Vulva. Auf 16 Junge beobachtete ich nur einmal, dass die Mutter mit dem Gebiss das Junge mit einem Ruck aus der Vulva zog. Das Junge . befand sich nur noch mit dem Kompf im mütterlichen Körper (Geburt in Steissendlage), als die Mutter es mit den Zähnen fasste. In den übrigen 15 Fällen leckten die Mütter die zum Teil geborenen Jungen, assen die Fruchthüllen, die den Jungen anklebten, auf, aber zogen die Jungen niemals heraus. So bald wie möglich versucht die Mutter mit der Zunge hinter die Nabelschnur zu kommen und diese mit dem Gebiss zu greifen. Dann zieht sie die Nachgeburt heraus und verzehrt diese so geschwind, dass. man nur ausnahmsweise etwas von der Plazenta sehen kann. Gelegentlich zerreist die Nabelschnur schon im mütterlichen Körper. In diesen Fällen wird die Plazenta nach dem Jungen ausgetrieben und sofort von der Mutter gefressen. In den normalen Fällen wird die Nabelschnur vom Muttertier durchgebissen. Sie bringt die Nabelschnur mit der Zunge zwischen die Backenzähne und zerkaut sie in möglichst kurzem Abstand vom Nabel des Jungen. Zwischen den Backenzähnen der Mutter und dem Bauch des Jungen befinden sich die Lippen des Muttertieres. Deshalb wird die Nabelschnur immer in der gleichen Entfernung (etwa $2 \mathrm{~mm}$ ) vom Nabel durchbissen, und sind die Nabelschnurreste, von einzelnen Ausnahmen abgesehen, immer gleich lang, was also bestimmt nicht durch eine praeformierte Rissstelle hervorgerufen wird. Schon wenn die Fruchthüllen zerrissen sind, aber das Junge nur zum Teil aus der Vulva hängt, bewegen und quäken und piepsen die Jungen, was nach MurR (1932) nicht der Fall sein sollte. Die Jungen sind spärlich weiss behaart, haben geschlossene Augen und sind etwa $6,5 \mathrm{~cm}$ lang.
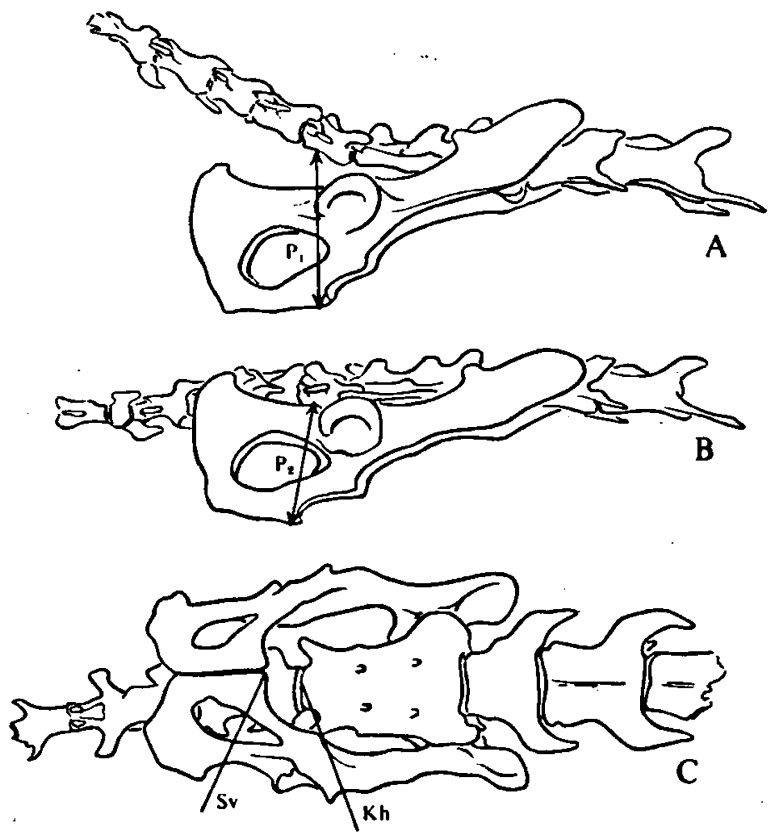

Abb. 5. Das knöcherne Becken des weiblichen Frettchens. A. Schwanz nach oben gerichtet, $\overline{\mathrm{P}_{1}}=$ Pectenverticale. B. Schwanz in normaler Haltung, $\mathrm{P}_{2}=$ Pectenverticale. Man beachte den grossen Grösseunterschied zwischen $P_{1}$ und $P_{2}$. C. Ventralansicht. Sv $=$ Symphysevorderseite. $\mathrm{Kh}=$ Kreuzbeinhinterrand. 
Die Jungen kommen entweder in Kopfendlage oder Steissendlage zur Welt. Auf 16 Junge fand ich 4 Steissendlagen, 11 Kopfenlagen und eine nicht genau zu bestimmende Lage (wahrscheinlich Kopfendlage), also 25\% Steissendlagen und 75\% Kopfendlagen. Von einem Alternieren von Kopf- und Steissendlagen (MURR, 1932) ist bestimmt nicht die Rede (Tabelle 1). Herter (1953) beobachtete nur 6 Kopfendlagen und keine Steissgeburten beim Iltis. Man würde 50\% Kopfendlagen und 50\% Steissendlagen erwarten (DE Snoo, 1947; NaAkTgeboren, 1960). Dies kann sehr gut richtig sein, denn 16 Junge ist viel zu wenig um einen zuverlässigen Eindruck dieser Prozentsätze zu bekommen. Ausser der Geburtslage ist auch die Haltung des Jungen während der Geburt von Bedeutung.

Die Jungen kommen bei der Kopfendgeburt immer in Beugehaltung zur Welt, d.h. der Kopf liegt der Brust eng an (Abb. 6A). In Kopfendlage tritt also zuerst der Nacken und das Hinterhaupt in der Vulva in Erscheinung (Abb. 6B). Bei einer Geburt in Steissendlage tritt das Steissende des Jungen zuerst aus der

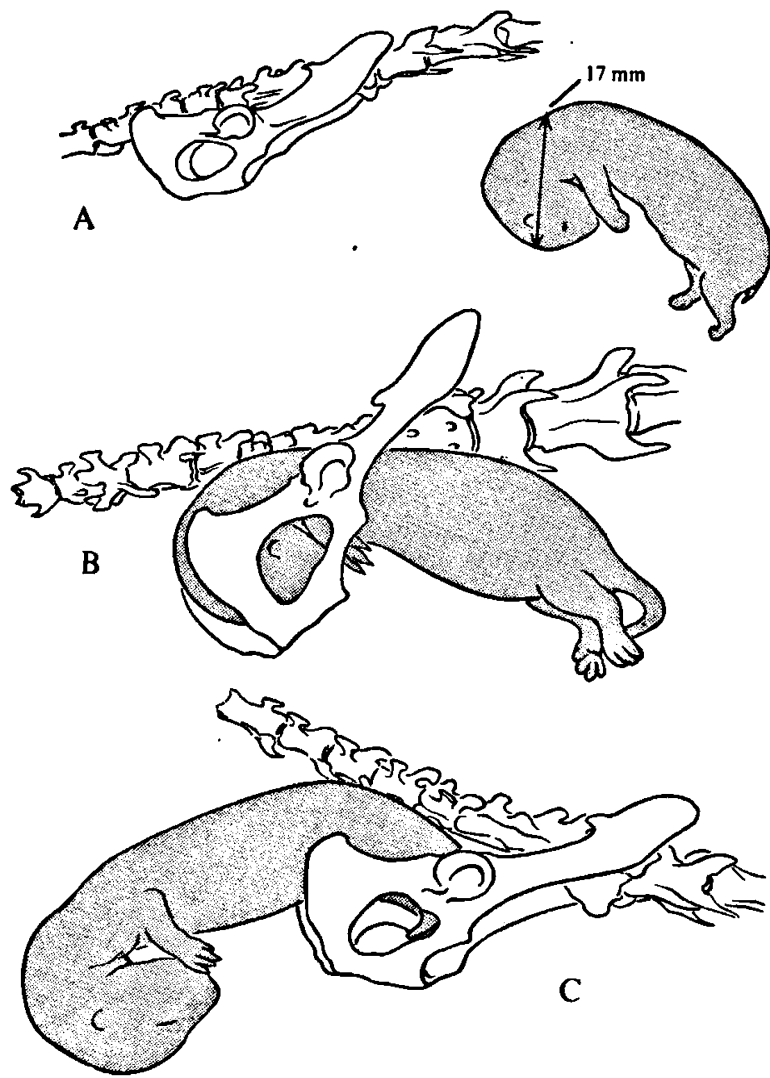

Abb. 6. Darstellung des Geburtsmechanismus in Kopfendlage am knöchernen Becken, nach Versuchen mit einem totgeborenen Jungen. Man beachte auch die Drehung vom Ilium im Iliosakralgelenk (vergleiche $A$ und $B$ ).
Vulva (Abb. 7 B, C). Das Steissende ist aber wenigstens während der Austreibung nicht gebogen. Deshalb ist bei normaler Haltung des Jungen der Umfang des Kopfendes viel grösser als jener der Steissendes. Bei einer Geburt in Steissendlage wird das Junge gänzlich gestreckt ausgetrieben, weil der Hals, wahrscheinlich beim Eintritt in das mütterlichen Becken, gestreckt wird (Abb. 7D). Diese Haltungsunterschiede beeinflussen die Austreibedauer pro Frucht. Tabelle 1 zeigt die Dauer der Austreibung pro Frucht und die Lage ( $\mathrm{K}=\mathrm{Kopf}-, \mathrm{S}=$ Steissendlage).

\section{TABELLE 1}

\begin{tabular}{|c|c|c|}
\hline $\begin{array}{l}\text { Nummer } \\
\text { des Jungen }\end{array}$ & Muttertier II & Muttertier III \\
\hline $\begin{array}{l}1 . \\
2 . \\
3 . \\
4 . \\
5 . \\
6 . \\
7 . \\
8 . \\
9 .\end{array}$ & 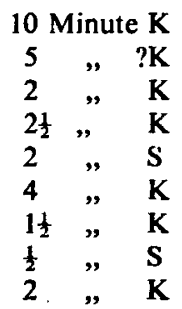 & $\begin{array}{lll}4 & \text { Minute } & \mathrm{K} \\
1 & & \mathrm{~K} \\
2 & \# & \mathrm{~K} \\
? & \# & \mathrm{~K} \\
1 & \# & \mathrm{~K} \\
5 & \text { Sekunde } & \text { S } \\
8 & \text { " } & \mathrm{S}\end{array}$ \\
\hline
\end{tabular}

Das erste Junge nimmt mehr Zeit als alle anderen Jungen in Anspruch. Dies lässt sich leicht verstehen, denn bei der Austreibung der ersten Jungen müssen die Geschlechtsteile der Mutter am stärksten ausgedehnt werden. Der Mittelwert der Austreibedauer in Kopfendlage beträgt 3,5 Minute pro Frucht, oder wenn man von den erstgeborenen Jungen absieht 2,6 Minuten. Der Mittelwert der Austreibedauer in Steissendlage beträgt nur 43 Sekunden pro Frucht. Es unterliegt keinem Zweifel, dass diese grossen Unterschiede durch die Haltungsunterschiede hervorgerufen werden. Der Kopf des Jungen ist $11 \mathrm{~mm}$ hoch und die gesammte Höhe des der Brust anliegenden Kopfes und der Brust beträgt $17 \mathrm{~mm}$. Es versteht sich also dass der Durchtritt in Kopfendlage (Abb. 6) schwieriger sein wird als in Streissendlage (Abb. 7), denn der Diameter verticalis oder Pectenverticale (Abb. 5B- $\mathrm{P}_{2}$ ) des mütterlichen Beckens ist bei normaler Haltung des Schwanzes nur 10,5 mm. Die Pectenverticale kann aber eine beträchtliche Vergrösserung erfahren, indem das Tier den Schwanz nach oben richtet $(\mathrm{Abb} \text {. 5A-P })_{1}$, denn die vordere Seite des Symphyse (Sv) findet sich beim Frettchen caudal des hinteren Kreuzbeinrandes (Kh, Abb. 5C). Wenn dies nicht der Fall wäre, so würde eine Geburt in Kopfendlage bei Beugehaltung des Jungen, zu den 


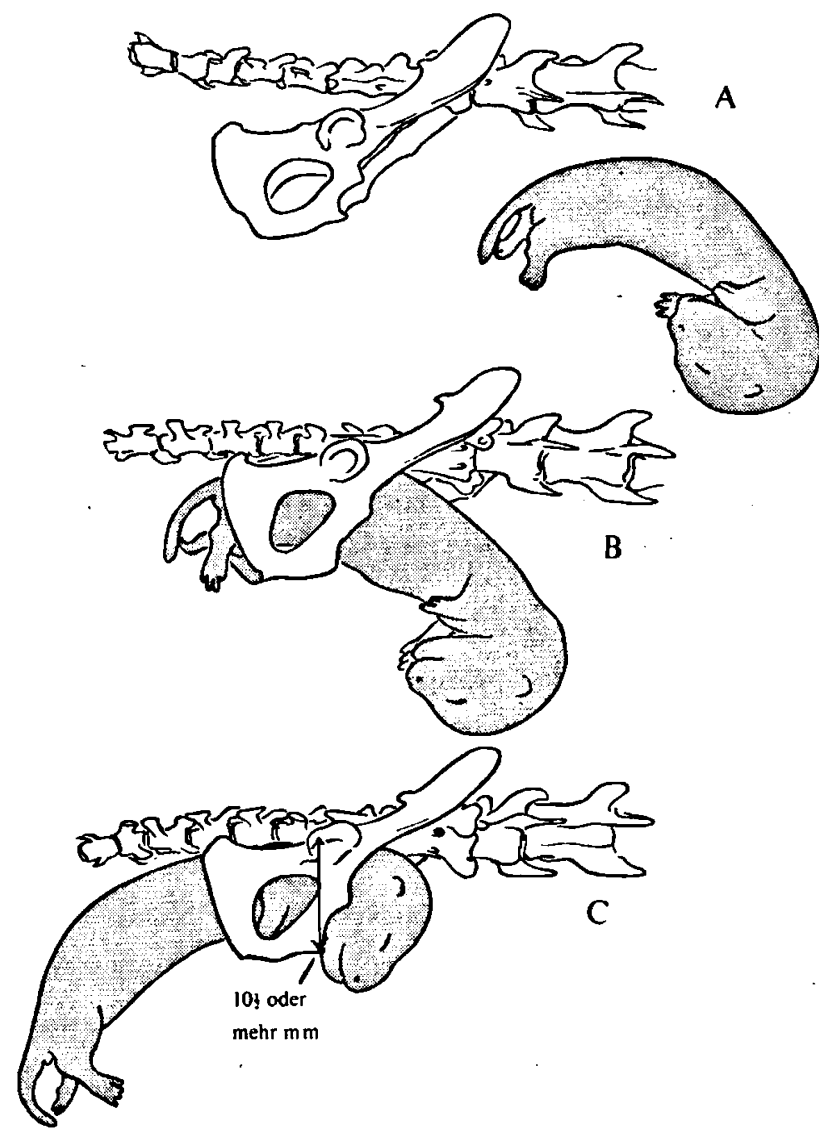

Unmöglichkeiten gehören, trotz des Umstandes, dass alle anderen Beckenabmessungen genügend gross sind, um das Junge ohne Schwierigkeiten passieren zu lassen. Stoss (1944) teilt mit dass auch für die Huftiere die Pectenverticale „für die Bestimmung der Geräumigkeit des Beckens von grösster Bedeutung ist". Murr (1932) fand 5 bis 10 Minuten für die Austreibung pro Frucht.

Die gesammte Austreibedauer war $2^{1 / 3}$ Stunden (Tier I, 12 Junge), 2 Stunden 37 Minuten (Tier II, 9 Junge) und 3 Stunden 8 Minuten (Tier III, 7 Junge). Diese Zeit ist gerechnet vom Austritt der ersten Fruchtblase aus der Vulva bis zur vollendeten Austreibung des letzten Jungen. Nach MurR (1932) ist die Austreibedauer etwa 3 Stunden, und Herter (1953) fand beim Iltis mehr als 2 Stunden. Zwischen den Geburten zweier Jungen stellte MurR Zeitabschnitte von einer Viertelstunde bis einer halben Stunde fest und Herter fand 10 Minuten bis höchstens 66 Minuten. In Tabelle 2 sind meine Beobachtungen dargestellt. Es gibt also eine Variationsbreitte von 3 Minuten bis 74 Minuten. Diese Zeitabschnitte sind gerechnet von der vollendeten Geburt eines Jungen bis zum Austritt der nächsten Fruchtblase aus der Vulva.

TABELLE 2

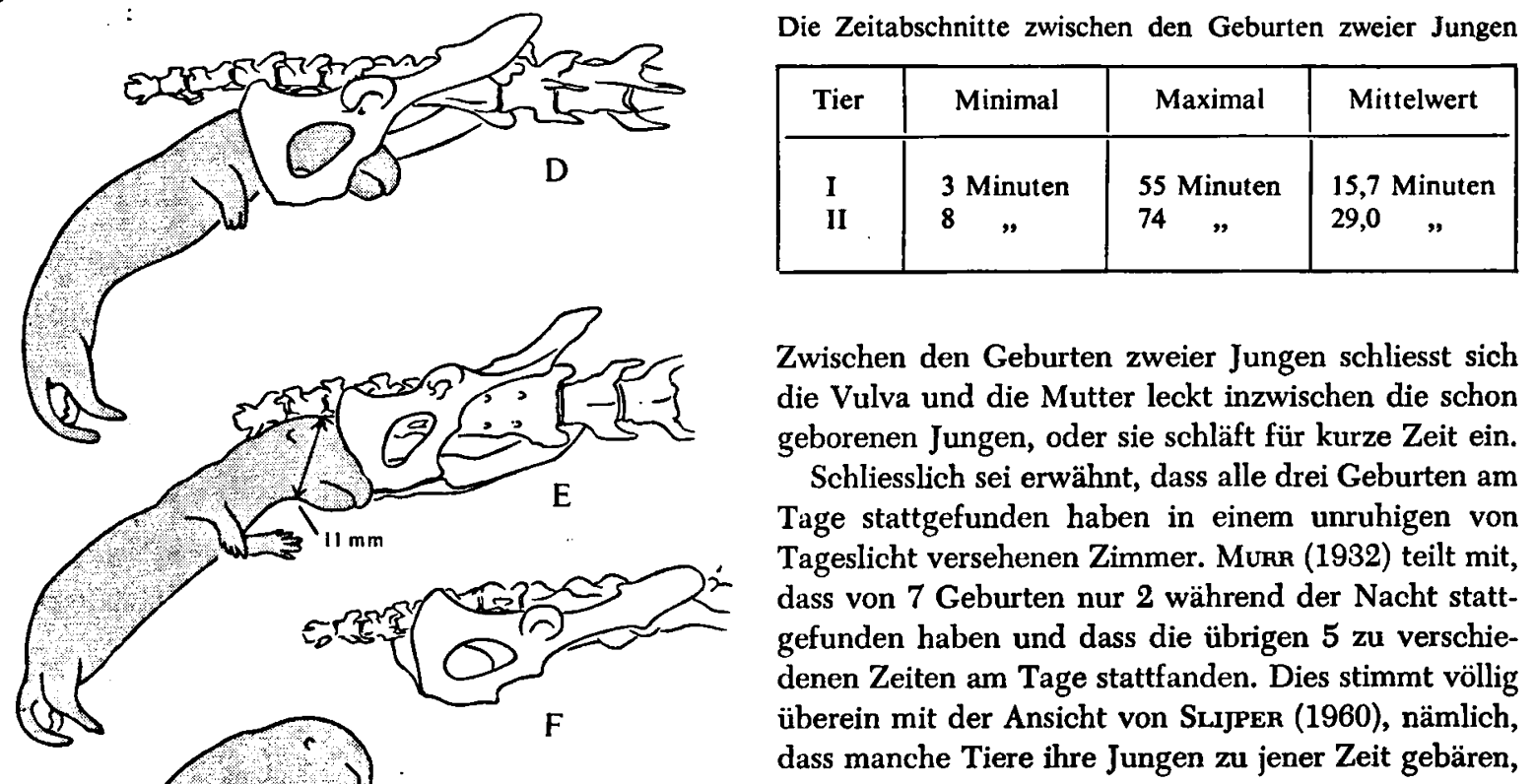

Abb. 7. Darstellung des Geburtsmechanismus in Steissendlage am knöchernen Becken, nach Versuchen mit einem totgeborenen Jungen. In den Teilabbildungen ist die Position des Betrachtens nicht immer die gleiche. 
in der sie zu schlafen gewohn sind. Von der Mardergruppe wissen wir, dass diese Tiere tatsächlich in der Nacht aktiv sind. Auch die Ratte wirft ihre Jungen meistens am Tage (NAAKTgeboren, 1960).

\section{Die Mutter und ihre Jungen nach der Geburt}

Nach der Geburt des letzten Jungen legt sich die ermüdete Mutter neben oder auf ihre Jungen (Abb. 4). Bald schläft sie ein. Man bekommt den Eindruck, dass das Muttertier ganz erschöpft ist. Tier III trank fleissig Milch nach der Geburt. Der Hinterkörper ist stark eingefallen und schleppt beim Gehen über den Boden, aber schon nach etwa 2 Tagen ist dies nicht mehr der Fall. Am ersten Tag nach der Geburt sind die Tiere schon sehr agressiv, was der Verfasser schmerzlich erfahren hat.

Von den 16 Jungen, die am 1. Juni geboren wurden, waren schon am nächsten Tag 2 gestorben. Von den übrigen 14 wurden jeden Tag die Nabelschnurreste untersucht. Der bald eingetrocknete Nabelschnurrest fällt nach etwa 4 bis 5 Tagen ab (Tabelle 3).

\section{TABelle 3}

\begin{tabular}{|c|c|c|}
\hline Datum & $\begin{array}{c}\text { Zahl der Jungen } \\
\text { mit } \\
\text { Nabelschnurrest }\end{array}$ & $\begin{array}{c}\text { Zahl der Jungen } \\
\text { ohne } \\
\text { Nabelschnurrest }\end{array}$ \\
\hline $4-6-1960$ & 14 & 0 \\
$5-6-1960$ & 9 & 5 \\
$6-6-1960$ & 4 & 10 \\
$7-6-1960$ & 0 & 14 \\
\hline
\end{tabular}

Die Jungen wachsen besonders schnell. Genauere Daten und Beschreibungen des Wachstums der Neugeborenen werden von Herter $(1953,1959)$ mitgeteilt.

\section{PROTOKOLLEN}

Frettchen-Geburt I, pluripar 24-5-1960

23-5-60 Die Fresslust ist schon seit einigen Tagen geringer als bei den beiden anderen Tieren.

16.30 Das Tier liegt im Schlafraum auf dem Rücken. $\mathrm{Ab}$ und zu erbebt der ganze Körper. Einmal liess das Tier ein stöhnendes Bellen, wie das eines Hundes, hören. Eben erhebt sich das Tier und schläft dann ein.

23.30 Das Tier schläft, nichts Besonderes geschieht.

24-5-'60

9.15 Lecken der Genitalia, Wehen?

10.15 Das Tier ist ruhig, nichts Besonderes.

12.15 Ziemlich weit geöffnete Vulva, etwas Schleimabsonderung. Symphysis pelvis normal, kein Zwischenraum zu fühlen.
13.15 Aus der Vulva tritt ein etwa $6 \mathrm{~mm}$ langer und $1,5 \mathrm{~mm}$ dicker Schleimfaden aus. Kein Blut im Schleim.

13.25 Das Tier frisst.

Lecken der Genitalia, danach auch Lecken des Bauches, der Zitzen und der gesamten Leistengegend. Das Tier liegt in stark gekrümmter Haltung auf dem Rücken.

15.28 Pressen. Flanken sehr eingezogen. Danach liegt das Tier sehr ruhig. Flanken beben. Das Beben der Flanken ist an sich nichts Besonderes, da das Tier an seinem ganzen Körper bebt, wenn es aus seinem Käfig genommen wird oder bei anderen schockenden Ereignissen.

15.40 Defaezieren im Tageskäfig.

16.15 Lecken der Genitalia. Das Tier liegt auf der Flanke und macht während des Leckens eine ganze Drehung. Dann wieder sehr ruhig.

17.15 Nichts Besonderes. Das Tier liegt ruhig und keucht.

19.4512 Junge geboren, davon eines tot. (Am nächsten Morgen 9 lebende und drei tote) Frisches Blut an der Vulva wurde kurz darauf abgeleckt. Alle Placentas waren verschwunden, also aufgefressen. Die Jungen waren spärlich behaart und an den meisten hing noch ein sehr kleiner Nabelschnurrest, bei einem war er $2 \mathrm{~cm}$ lang. Die Geburt kann höchstens 21/2 Stunde gedauert haben.

26-5-'60 Das sonst immer sehr ruhige und zahme Muttertier, das niemals biss, ist nun sehr agressiv und bissig.

\section{Frettchen-Geburt II, primipar 1-6-1960}

31-5-'6ó Das Tier ist sehr ruhig, starker Haarausfall.

17.00 Vulva mässig geschwollen. Das Tier liegt regelmässig auf dem Rücken und scheint ab und zu Wehen zu haben.

20.30 In die Hand genommen, war starkes Zittern des Abdomens zu fühlen: Wehen. Zittern nimmt wieder $a b$ und kehrt innerhalb einer Viertelstunde nicht wieder zuruick. Das Tier schläft ein.

23.00 bis 1-6.' $60,01.00$

Ab und zu erwacht das Tier und leckt Bauch und Zitzen. Lecken der Genitalia nicht wahrgenommen. Vulva geschwollen. Das Tier schläft viel.

$1-6-60$

12.00 Aus der Vulva kommt ein $1,5 \mathrm{~cm}$ langer, $1 \mathrm{~mm}$ dicker, weisser Schleimfaden. Das Tier ist ruhig und liegt häufig auf dem Rücken. Es legte sich im Tageskäfig sogar auf den Bauch, was es sonst nie tat. Träge.

12.02 Urinieren, Defaezieren.

12.06 Das Tier trinkt nicht wie gewöhnlich stehend, sondern liegend. Nach dem Trinken bleibt es noch eben auf dem Bauch liegen. Die Flanke weist deutlich zitternde, nicht mit der Atmung zusammenhängende Bewegungen auf.

12.08 Trinken. Danach geht das Tier in den Schlafraum. Es ist träge und reckt sich mit hohlem Rückken. Es scheint, dass es ihm schwer fällt, auf seinen Hinterbeinen zu stehen. Das Tier legt sich im Schlafraum auf den Rücken. Schockende Bewegungen im Abdomen. 
Urinieren, wobei weisser Schleim ausgeschieden wird. Dann legt sich das Tier im Schlafraum auf den Bauch.

12.37 Recken und Rollen.

13.20 Noch immer kommt Schleim aus der Vulva, die nun $1-1,5 \mathrm{~mm}$ geöffnet ist.

13.45 Das Tier schläft ruhig.

14.10 Das Tier geht in die Kotecke im Tageskäfig und drückt als wolle es defaezieren. Es kommt nichts. Das Tier knickt durch die Hinterbeine. Pressen mit stark eingefallenen Flanken und durchgebogenem Rücken. Noch eben bleibt das Tier liegen und geht in den Schlafraum. Gleich darauf kehrt es zur Kotecke zurück. Heftiges Pressen. Die Vulva dehnt sich.

14.18 In der Vulva erscheint eine bräunliche-gelbe Fruchtblase. Das Tier läuft nun zwischen Tageskäfig und Schlafraum hin und her.

14.20 Pressen. Der Kopf des ersten Jungen erscheint. Die Fruchthäute sind gerissen. Das Junge bewegt sich und öffnet sein Maul. Das Muttertier geht in den Tageskäfig.

14.22 Pressen, die Vorderbeine kommen zum Vorschein. Lecken.

14.25 Im Schlaufraum anhaltendes Lecken des halb geborenen Jungen in gebogener Haltung, auf der Flanke liegend.

14.26 Nur noch die Hinterbeine befinden sich in der Vulva. Das Muttertier leckt das Junge.

14.28 Erstes Junge in Kopfendlage geboren. Das Junge piept und wird von der Mutter geleckt. Lecken der Vulva. Nachgeburt aufgefressen?

14.30 Das Muttertier leckt das Junge, nimmt es in sein Maul und legt es an eine andere Stelle.

14.34 Lecken der Vulva.

14.35 Pressen und Lecken der Vulva, liegend und drehend auf der Flanke.

14.41 Lecken des Jungen.

14.42 Pressen mit durchbeugendem Körper, zuckende Flanken. Wehen. Pressen und Lecken der Vulva.

14.45 Pressen und Lecken. Die Fruchtblase erscheint in der Vulva. Tier läuft in den Tageskäfig, kommt gleich wieder zurück.

14.46 Muttertier leckt und presst, dabei kommt die Fruchtblase weiter aus der Vulva. Dann nimmt sie mit dem Maul das erste Junge auf und presst.

14.48 Tier presst in der Kotecke in Defaekationshaltung, geht zurück in den Schlafraum.

14.49 Lecken der Vulva und der Fruchtblase.

14.50 Das zweite Junge geboren. Lage unsicher, wahrscheinlich Kopfendlage. Das Muttertier leckt sich und leckt das zweite Junge.

14.54 Lecken der Vulva. Beide Junge vollständig gesäubert, sie piepen und bewegen sich.

14.57 Fruchtblase erscheint in der Vulva, kommt schnell weiter heraus. Das Tier begibt sich in den Tageskäfig. Kopf des Jungen zu sehen. Das Muttertier leckt und frisst die Fruchthäute.

14.59 Das dritte Junge geboren in Kopfendlage. Placenta gefressen. Viel Blut an der Vulva.

15.00 Lecken des Jungen.

15.01 Lecken der Vulva.

15.04 Lecken der Vulva und der Leistengegend.
15.05 Begibt sich in den Tageskäfig und kommt zurïck.

15.07 Lecken der Vulva, dann Aufsuchen der Kotecke.

15.10 Defaezieren. Blutaustritt aus der Vulva. Dann läuft es in den Schlafraum, kratzt am Boden, legt sich und leckt die Jungen.

15.15 Das Tier liegt ruhig und leckt die Jungen.

15.17 Lecken der Vulva.

15.21 Das Muttertier schläft.

15.25 Lecken der Vulva und der Jungen.

15.31 Lecken der Vulva.

15.35 Lecken der Vulva, dabei drehen im Nest. Der Schwanz wird immer nach oben gehalten.

15.38 Wehen.

15.40 Das Tier liegt auf der Flanke und dehnt den Unterkörper. Schlafen.

15.45 Kräftiges Dehnen, fast in Rückenlage. Der Kopf der beiden bei der Vulva liegenden Jungen ist nass. Blasensprung?

15.48 Pressen und Lecken der Jungen.

15.50 Lecken der Vulva und pressen in Flankenlage.

15.54 Geburt des vierten Jungen in Kopfendlage, während das Muttertier läuft.

15.55 Geburt des vierten Jungen fast beendet. Das Muttertier leckt Vulva und Junge.

15.57 Das Junge liegt immer noch mit dem Schwanze in der Vulva und mit dem Kopf bei den Zitzen.

15.58 Das Muttertier zieht die Nachgeburt aus der Vulva und frisst sie sogleich auf.

16.00 Wehen, pressen, Fruchtblase sichtbar. Das Muttertier steht auf, das Junge sogleich etwa zur Hälfte aus der Vulva. Tier geht in den Tageskäfig. Lecken und Zurücklaufen. Nabelschnur mit den Backenzähnen abgebissen.

16.02 Das fünfte Junge in Steissendlage geboren. Muttertier leckt das Junge.

16.03 Pressen. Lecken der Leistengegend und der Vulva.

16.05 Pressen und Lecken der Vulva, das sie regelmässig wiederholt. Beim Pressen kam etwas rote Flüssigkeit aus der Vulva.

16.12 Lecken der Vulva. Einige Tropfen gelb-rote Flüssigkeit.

16.15 Lecken der Vulva, sich dabei immer etwas umdrehend. Auch Lecken des Bauches, der Oberschenkel und der Jungen. Schlafversuch. Ab und zu Pressen.

16.20 Wieder kommt etwas Blut aus der Vulva.

16.22 Defaezieren. Aus der Vulva keinerlei Absonderungen. Lecken der Vulva.

16.25 Pressen. Das Tier begibt sich in den Tageskäfig. Plötzlich das sechste Junge in Kopfendlage halb aus der Vulva. Blasensprung erfolgt. Tier läuft mit dem aus der Vulva hängenden Jungen hin und her.

16.28 Lecken des Jungen und der Vulva.

16.30 Geburt des sechsten Jungen beendet. Nabelschnurrest etwa $2 \mathrm{~mm}$ lang.

16.37 Lecken der Vulva.

16.40 Das Tier liegt ruhig. Dann im Liegen heftiges Pressen. In der Vulva erscheint eine Fruchtblase. Mit jeder Wehe kommt sie weiter heraus. Das Muttertier frisst die Fruchthäute ab. Kopf des siebenten Jungen zu sehen. Innerhalb einer an- 
derthalben Minute bis an die Schenkel ausgetrieben. Kopfendlage.

16.44 Durchbeissen der Nabelschnur.

16.45 Lecken der Vulva und der Oberschenkel.

16.47 Kräftiges Pressen. Fruchtblase aus der Vulva. Das achte Junge innerhalb von 30 Sekunden in Steissendlage ausgetrieben. Die Fruchthäute wurden erst nach der Austreibung abgefressen. Viel Blut an Oberschenkeln und Vulva des Muttertieres. Während der Geburt des achten Jungen lag das Muttertier auf der Flanke im Schlafraum.

16.49

16.50

16.54

16.55

Lecken der Vulva und der Leistengegend.

Lecken der Vulva.

Lecken der Vulva.

Das neunte Junge in Kopfendlage gleich bis zur Hälfte ausgetrieben. Das Muttertier frisst die Fruchthäute $a b$. Mit den Zähnen zieht sie mit der Nabelschnur die Placenta heraus, frisst sie gleich auf und beisst die Nabelschnur durch. Die Mutter blicb im Schlafraum, die Austreibungsperiode betrug weniger als eine Minute.

16.57 An Oberschenkeln und Vulva viel Blut, Muttertier leckt es ab.

17.00

17.05

17.08

17.25

17.28

Lecken der Oberschenkel und der Vulva.

Lecken der Vulva.

Mutter liegt auf der Flanke und leckt die Jungen, derer einige schon die Zitzen suchen.

Dasgleiche.

Urinieren, Defaezieren, Vulva lecken. Dann liegt das Tier wieder sehr ruhig.

\section{Frettchen-Geburt III, pluripar 1-6-1960}

Dieses Tier unterschied sich in keinerlei Hinsicht von dem Tier der Frettchen-Geburt II. Es war gleichfalls sehr ruhig, enthaarte sich jedoch fast nicht.

1-6-'60 Geringere Fresslust.

15.10 Unruhiges Hin- und Herlaufen. Liegen im Tageskäfig. Pressen in der Kotecke.

15.20 Geburt des ersten Jungen in Kopfendlage.

Lecken des Jungen.

15.24 Lecken der Vulva.

15.28 Placenta noch im Inneren des Muttertieres, Nabelschnur noch unversehrt.

15.29 Nabelschnurrest des Jungen etwa $10 \mathrm{~mm}$ lang. Placenta gefressen. Nabelschnurrest mit den Backenzähnen bis auf $2 \mathrm{~mm}$ vom Nabel entfernt abgebissen.

15.32 Lecken der Vulva und der Oberschenkel.

15.34

15.35

15.37

15.38

15.41

15.43

15.44

15.48

15.51

Lecken des Jungen.

Lecken des Jungen.

Muttertier fasst ihr Junges mit den Zähnen.

Seit 15.32 unregelmässig Strecken der Hinter-

beine und schwaches Pressen. Nun ab und zu Einziehen der Flanken.

Pressen.

Kratzen auf dem Boden des Schlafraumes.

Lecken der Vulva und Pressen.

Wehen. Kurzer Schlaf in eingerollter Haltung.

Lecken der Vulva. Ab und zu Pressen und Einziehen der Flanken. Das Tier lässt Röcheltöne hören. Sehr schnell hintereinander Pressen.

Lecken der Vulva.
Pressen in der Kotecke. Das Tier liegt flach am Boden. Fruchtblase erscheint in der Vulva, das Tier geht in den Schlafraum. Das Junge kommt in Kopfendlage zum Vorschein. Das Muttertier leckt das Junge. Dauer der Austreibungsphase eine Minute.

15.59 Das Junge ist bis zum Becken geboren. Das Junge atmet. Das Muttertier leckt die Vulva und das Junge. Keine Fruchthautreste mehr am Jungen.

16.02 Lecken der Vulva und des Jungen.

16.05 Lecken der Oberschenkel, der Vulva und der Jungen.

16.08 Pressen.

16.09 Pressen und Lecken der Vulva.

16.11 Lecken der Vulva.

16.15 Lecken der Vulva. Das Tier legt sich auf seine Flanke und leckt die Jungen.

16.20 Das Tier schläft.

16.28 Wehen während des Schlafens. Pressen.

16.30 Aus der Vulva Flüssigkeitsabsonderung. Lecken der Vulva und Auflecken der Flüssigkeit vom Boden.

16.33 Lecken der Vulva.

16.35 Lecken der Vulva.

16.36 Das Tier steht auf und läuft durch den Tageskäfig. Nach dem Defaezieren zurück in den Schlafraum.

16.39 Lecken der Vulva.

16.40 Lecken der Jungen.

16.45 Das Tier liegt ruhig.

16.50 Schlafen. Blut in der Vulva.

16.52 Pressen, Blut aus der Vulva.

16.57 Pressen, Lecken der Vulva.

17.01 Das Muttertier hat ein Junges im Maul, sucht die Vulva und dreht sich dabei im Kreise herum.

17.05 Lecken der Vulva.

17.13 Heftiges Pressen in Flankenlage. Lecken der Vulva.

17.16 Sehr heftiges Pressen im Liegen. Das dritte Junge erscheint in Kopfendlage in der Fruchtblase. Das Muttertier leckt das Junge. Dauer der Austreibungsphase zwei Minuten.

17.27 Geburt des vierten Jungen in Kopfendlage. Nabelschnurrest etwa $4 \mathrm{~mm}$. Während der Geburt des dritten und vierten Jungen, verliess das Muttertier den Schlafraum nicht. Die Geburten geschahen in Beugehaltung. Der Kopf der Jungen lag der Brust an. Das Genick und der Hinterkopf erschienen zuerst.

17.30 Langanhaltendes Lecken der Vulva.
17.32 Placenta ausgetrieben.

17.33 Fressen der Placenta und Lecken der blutbeschmierten Vulva und Oberschenkel. Die Vulva ist nicht mehr geschwollen und wieder geschlossen.

17.40 Lecken der Vulva und des Jungen.

17.45 Pressen. Defaezieren in der Kotecke, zurück in den Schlafraum.

17.49 Röcheltöne. Pressen.

17.52 Das fünfte Junge in Kopfendlage sehr schnell geboren. Lecken. Kurze Unterbrechung, als das Junge etwa bis zum Nabel ausgetrieben war.

18.00 Das Muttertier beisst die Nabelschnur durch und 
leckt die Nachgeburt aus der Vulva. Pressen. Das sechste Junge in Steissendlage innerhalb von fünf Sekunden ausgetrieben. Ablecken des Jungen bis es sauber und trocken ist.

18.02 Lecken der Vulva.

18.05 Blut in der Vulva. Lecken der Vulva und der Leistengegend.

18.09 Pressen. Während eintger Zeit liegt das Tier ziemlich ruhig.

18.22 Pressen und Röcheltöne.

18.23 Das siebente Junge in Steissendlage innerhalb von acht Sekunden ausgetrieben. Die Fruchthäute sind unversehrt. Als der Kopf des Jungen in der Vagina erschienen war, fastte das Muttertier das Junge mit den Zähnen und zog es heraus.

18.28 Nabelschnur etwa $4 \mathrm{~mm}$ vom Nabel entfernt durchgebissen. Lecken der Vulva.

18.29 Pressen und Lecken der Vulva. Aus der Vulva kommt Blut und danach eine ganze Placenta. Das Muttertier frisst diese sofort auf.

18.32 Lecken der Vulva und der Oberschenkel.

18.34 Lecken der Jungen.

18.37 Flanken sehr eingefallen. Das Tier trinkt begierig Milch. Beim Laufen schleift der Bauch über den Boden.

\section{ZUSAMMENFASSUNG}

Beschreibung der Geburt von drei Frettchen mit drei Protokollen. In mancher Hinsicht etwa die gleichen Befunde wie Murr (1932). Hier werden nur die abweichenden und neuen Befunde mitgeteilt.

1. Keine Unterschiede zwischen primiparen und pluriparen Tieren.

2. Eine glasige Schleimschnur aus der Vulva ist 2 bis 4 Stunden vor der Austreibung wahrnehmbar. Kurz vor der Geburt steht das Muttertier nur mit grösster Schwierigkeit und liegt oftmals auf dem Rücken.

3. Die Austreibung fängt an mit Pressen. Das Tier nimmt dazu die charakteristische Kothaltung an und geht in die Kotecke.

4. Nachdem die Fruchtblase in der Vulva er- schienen ist, wird sie vom Muttertier mit dem Gebiss zerrissen. Nur ausnahmsweise findet ein spontaner Blasensprung statt.

5. Das Junge wird im Liegen weiter ausgetrieben. Die Wehen werden von der Bauchpresse unterstützt, wobei die Mutter liegen bleibt. Nur einmal (auf 16 Junge) half die Mutter und zog mit dem Gebiss das Junge auf einmal aus der Vulva.

6. Die Nabelschnur wird von der Mutter mit den Backenzähnen durchbissen. Am Bauch des Jungen bleibt ein etwa $2 \mathrm{~mm}$ langer Rest übrig. Diese Länge entspricht genau der Lippendicke des Muttertieres, also der Entfernung ihrer Backenzähne vom Nabel des Neugeborenen.

7. Die Nachgeburt wird unmittelbar nach ihrem Erscheinen gefressen, meistens schon bevor man in der Lage ist, diese genau zu beobachten.

8. Auf 16 Junge wurden nur 4 Steisslagen gefunden, also 25\%, Kopfendlagen und die Steissendlagen alternierten nicht.

9. In Kopfendlage wird das Junge in Beugehaltung geboren (Kopf liegt der Brust an), aber in Steissendlage ist das Junge gestreckt.

10. Der Mittelwert der Austreibezeit pro Frucht ist in Kopfendlage 3,5 Minuten, oder wenn man von dem erstgeborenen Jungen absieht, 2,6 Minuten, und in Steissendlage nur 43 Sekunden.

11. Zwischen den Geburten von 2 Jungen liegen Zeitabschnitte von 3 bis 74 Minuten.

12. Der Nabelschnurrest am Bauch des Jungen fällt ab nach 4 bis 5 Tagen.

\section{NACHTRAG}

Die oben beschriebene Befunde komte ich im Juni 1961 bestätigen. In einen wurf wurden acht Jungen geboren: sieben in Kopfendlage und eines unsichere Lage. Beide Geburten fanden am Tage statt. Die Vorzeichen der Geburt beim Mink (Mustela vison) sind genau dieselbe wie beim Frettchen. Leider konnte ich die Geburt beim Mink nicht beobachten.

\section{LITERATUR}

Granzow, J., 1930: Zur vergleichenden Physiologie der Geburtsvorgänge. Arch. f. Gynäkologie, 139, 317-340. Henter, K., 1953: Uber das Verhalten von Iltissen. Zeitschr. f. Tierpsych. 10, 56-71, besonders 60-61.

Herter, K., 1959: Iltisse und Frettchen. 1-112, besonders 93 und 96. Neue Brehm Bücherei, Heft 230. Ziemsen Verlag, Wittenberg Lutherstadt.

MURR, E., 1932: Beobachtungen über den Geburtsvorgang beim Frettchen. Zool. Garten (N.F.) 5, 37-40.

MurR, E., 1933: Aus der Fortpfanzungsbiologie des Frettchens (Putorius furo L.). Zeitschr. f. Säugetierkunde 8, 26-32.
NAAKTGEBOREN, C., 1960: Enkele waarnemingen over de geboorte van de laboratoriumrat, Rattus norvegicus (Berkenhout). Lutra, 2, 23-30.

SlıJPER, E. J., 1960: Die Geburt der Säugetiere. Kükenthals Handbuch der Zoologie, Band 8, Lief. 25, 1-108. de Gruyter, Berlin.

SNOO, K. DE, 1947: Het probleem der Menschwording in het licht van de vergelijkende verloskunde. 1-320, Bohn, Haarlem. 2. Aufl.

Stoss, A. O., 1944: Tierärztliche Geburtskunde und Gynäkologie. 1-419, Enke, Stuttgart. 2. Aufl. 